\title{
A Novel CAM for the Luminance Levels in the Same Chromaticity Viewing Conditions
}

\author{
Soo-Wook Jang, Eun-Su Kim, Sung-Hak Lee, and Kyu-Ik Sohng \\ School of Electronic Engineering and Computer Science, \\ Kyungpook National University, \\ 1370, Sankyug-Dong, Buk-Gu, Daegu, 702-701, Korea \\ \{jjang, saeloum, shark2, kisohng\}@ee.knu.ac.kr
}

\begin{abstract}
In this paper, we propose the chromatic adaptation model (CAM) for the Variations of the Luminance Levels in the same Chromaticity Viewing Conditions. The proposed model is obtained by the transform the test colors of the high luminance into the corresponding colors of the low luminance. In the proposed model, the optimal coefficients are obtained from the corresponding colors data of the Breneman's experiments. In the experimental results, we confirmed that the chromaticity errors between the predicted colors by the proposed model and the corresponding colors of the Breneman's experiments are 0.004 in $u^{\prime} v^{\prime}$ chromaticity coordinates. The prediction performance of the proposed model is excellent because this error is the threshold value that two adjacent color patches can be distinguished.
\end{abstract}

\section{Introduction}

Color TV display systems have been designed and developed for the colorimetric and preferred color reproduction[1]. Preferred color reproduction is reproduction in which the colors depart from equality of appearance to those in the original in order to give a more pleasing result. This might be applicable in situations such as TV display in which consumers prefer to have pictures that reproduce colors closer to their memory colors for objects such as skin tones, foliage, sky, bodies of water, and so on. Colorimetric color reproduction in TV system is defined via metameric matches between the original and the reproduction such that they both have the same CIE $x y$ chromaticity coordinates. In this case, the reproduced luminance levels are not equal with the original[2]. Real surround viewing conditions in watching $\mathrm{TV}$ and $\mathrm{PC}$ monitor are quite different from the photographing conditions in various luminance levels and chromaticities of illuminants. Human visual system (HVS) is adapted chromatically under the different viewing conditions in luminance levels and chromaticities of illuminants. Accordingly, the reproduced colors of the same chromaticity will appear as quite different color. Therefore, it is necessary that the displayed colors are reproduced to be appeared as the original colors in the photographing conditions.

Chromatic adaptation is one of the human visual characteristic that physically different colors look like equal according to the surround viewing 
conditions[2]-[7]. In this case, C2 under any other surround viewing conditions is called a corresponding color which $\mathrm{C} 1$ looks exactly the same color under a certain viewing condition.

There are various models that can be used to reproduce corresponding colors considering the chromatic adaptation according to the change of surround illuminants' chromaticity under the same luminance conditions[2]-[11]. However, there is almost unknown about the chromatic adaptation for the variations of the luminance levels under the same illuminants. And only the human visual characteristic about the variations of the luminance levels had been studied[6][7], [12]. Also, the CAMs for such phenomenon have not been proposed yet. In this paper, we propose the chromatic adaptation model for the variations of the luminance levels under the same illuminants.

\section{The Proposed CAM}

Under the sunlight conditions which have high luminance, the correlated color temperature (CCT) of clear sky is one of the popular research field at present and is about $5500 \sim 6000 \mathrm{~K}[13]$. As shown in Fig. 1, the luminance level of white under the sunlight is about $10000 \mathrm{~cd} / \mathrm{m}^{2}$ and the luminance level of the living room is about $10 \mathrm{~cd} / \mathrm{m} 2$ in average[14]. Thus, real surround viewing conditions in watching TV and PC monitor are quite different from the photographing conditions in various luminance levels of illuminants. HVS is adapted chromatically under the different viewing conditions in luminance levels of illuminants. Accordingly, the reproduced colors of the same chromaticity will appear as quite different color.

In this paper, the CAM is proposed according to the change of luminance level under the same illuminants. In Fig. 1, the proposed model is obtained by the transform the test colors of the high luminance into the corresponding colors of the low luminance. The proposed CAM is expressed in Eqs.(1) through (5). The first step is a transformation from the CIE tristimulus values $X Y Z$ to the fundamental tristimulus $L M S$. The Hunt-Pointer-Estevez transformation with illuminant $\mathrm{D}_{65}$ normalization is used[2],[11].

$$
\begin{aligned}
& {\left[\begin{array}{c}
L_{1} \\
M_{1} \\
S_{1}
\end{array}\right]_{Y_{1}}=\mathbf{M}\left[\begin{array}{c}
X_{1} \\
Y_{1} \\
Z_{1}
\end{array}\right]_{Y_{1}}, \quad\left[\begin{array}{c}
L_{2} \\
M_{2} \\
S_{2}
\end{array}\right]_{Y_{2}}=\mathbf{M}\left[\begin{array}{c}
X_{2} \\
Y_{2} \\
Z_{2}
\end{array}\right]_{Y_{2}}} \\
& \mathbf{M}=\left[\begin{array}{rrr}
0.4002 & 0.7076 & -0.0808 \\
-0.2263 & 1.1653 & 0.0457 \\
0.0000 & 0.0000 & 0.9182
\end{array}\right]
\end{aligned}
$$

The next step is to apply the proposed model as shown in Eqs. $(3) \sim(5)$.

$$
\left[\begin{array}{c}
L_{2} \\
M_{2} \\
S_{2}
\end{array}\right]=\mathbf{M}_{\triangle Y}\left[\begin{array}{c}
L_{1} \\
M_{1} \\
S_{1}
\end{array}\right]=\left[M_{i j}\right]_{3 \times 3} \cdot\left[\begin{array}{c}
L_{1} \\
M_{1} \\
S_{1}
\end{array}\right]
$$




$$
\begin{aligned}
& M_{i j}=a_{i j}+b_{i j}\left(\frac{Y_{2}}{Y_{1}}\right)+c_{i j}\left(\frac{Y_{2}}{Y_{1}}\right), \quad\left(Y_{2} \geq Y_{1}\right) \\
& {\left[\begin{array}{c}
X_{2} \\
Y_{2} \\
Z_{2}
\end{array}\right]=\mathbf{M}^{-1} \mathbf{M}_{\triangle Y} \mathbf{M}\left[\begin{array}{c}
X_{1} \\
Y_{1} \\
Z_{1}
\end{array}\right]}
\end{aligned}
$$

In these equations, $Y_{1}$ and $Y_{2}$ are maximum white luminance under a certain surround viewing conditions, and $Y_{2}$ is higher than $Y_{1}$. And $\mathbf{M}_{\triangle Y}$ represents $3 \times 3$ transfer matrix for chromatic adaptation according to the variations of the luminance levels under the same illuminants and also each element of transfer matrix is modeled by maximum luminance ratio, $Y_{2} / Y_{1}$, in Eq. 4.

In the proposed model, the optimal coefficients of each element in transfer matrix are obtained from the corresponding colors data of the Breneman's experiments[7]. And these optimal coefficients and element functions are shown in Table 1. Because transfer matrix of the proposed model is the square matrix, the proposed model can be transformed easily from high luminance conditions to low luminance conditions using the inverse matrix. And once the surround viewing condition is determined, the proposed model has the coefficient of linear transformation and can be easily applied to the color display devices.

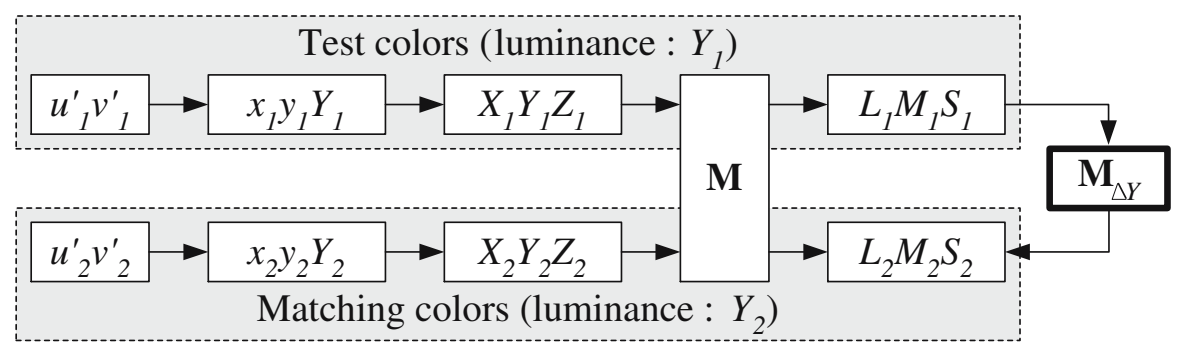

Fig. 1. Block diagram for the proposed model

Table 1. Element functions' coefficients of the transfer matrix $\mathbf{M}_{\triangle Y}$ of the proposed medel

\begin{tabular}{|c|c|c|c|}
\hline $\mathrm{M}_{\mathrm{ij}}$ & $\mathrm{a}_{\mathrm{ij}}$ & $\mathrm{b}_{\mathrm{ij}}$ & $\mathrm{c}_{\mathrm{ij}}$ \\
\hline $\mathrm{M}_{11}$ & 0.2254 & 0.7691 & 0.0056 \\
\hline $\mathrm{M}_{12}$ & -0.1966 & 0.1993 & -0.0026 \\
\hline $\mathrm{M}_{13}$ & -0.0186 & 0.0197 & -0.0012 \\
\hline $\mathrm{M}_{21}$ & -0.1275 & 0.1306 & -0.0031 \\
\hline $\mathrm{M}_{22}$ & 0.1112 & 0.8873 & 0.0015 \\
\hline $\mathrm{M}_{23}$ & 0.0104 & -0.0112 & 0.0007 \\
\hline $\mathrm{M}_{31}$ & 0.6355 & -0.6743 & 0.0388 \\
\hline $\mathrm{M}_{32}$ & -0.8223 & 0.8645 & -0.0422 \\
\hline $\mathrm{M}_{33}$ & 0.2089 & 0.7989 & -0.0077 \\
\hline
\end{tabular}




\section{$3 \quad$ Experiments and Results}

The performance of the proposed model is evaluated in terms of color fidelity. The chromaticity errors, $\triangle u^{\prime} v^{\prime}$, and color reproduction errors, $\triangle E_{u v}^{*}$, are expressed in Eqs. (6) through (7). Table 2 shows the chromaticity errors and color reproduction errors between the predicted colors by the proposed model and the corresponding colors of the Breneman's experiments.

$$
\begin{aligned}
& \triangle u^{\prime} v^{\prime}=\sqrt{\left(\triangle u^{\prime}\right)^{2}+\left(\triangle v^{\prime}\right)^{2}} \\
& \triangle E_{u v}^{*}=\sqrt{\left(\triangle L^{*}\right)^{2}+\left(\triangle u^{*}\right)^{2}+\left(\triangle v^{*}\right)^{2}}
\end{aligned}
$$

In the experimental results, we confirmed that the chromaticity errors between the predicted colors by the proposed model and the corresponding colors of the Breneman's experiments are 0.004 in a $u^{\prime} v^{\prime}$ chromaticity coordinates. It is known that two adjacent color patches can usually be distinguished with a $\triangle u^{\prime} v^{\prime} \geq 0.004$, but for separated colors, a shift $\triangle u^{\prime} v^{\prime} \geq 0.04$ is often required to notice a color change in the $u^{\prime} v^{\prime}$ chromaticity coordinates system[1]. Therefore, the prediction performance of the proposed model is excellent because this errors are the threshold value that two adjacent color patches can be distinguished.

\begin{tabular}{|c|c|c|c|c|c|c|}
\hline \multirow{2}{*}{$\mathrm{M}_{\mathrm{ij}}$} & \multicolumn{4}{|c|}{ Luminance of white } & \multirow{2}{*}{\multicolumn{2}{|c|}{850 to $11100 \mathrm{~cd} / \mathrm{m}^{2}$}} \\
\hline & 15 to 2 & $0 \mathrm{~cd} / \mathrm{m}^{2}$ & 130 to 2 & $20 \mathrm{~cd} / \mathrm{m}^{2}$ & & \\
\hline Sample colors & $\Delta u^{\prime} v^{\prime}$ & $\Delta E_{u v}^{*}$ & $\Delta u^{\prime} v^{\prime}$ & $\Delta E_{u v}^{*}$ & $\Delta u^{\prime} v^{\prime}$ & $\Delta E_{u v}^{*}$ \\
\hline Gray & 0.002133 & 1.670525 & 0.001905 & 1.492598 & 0.005212 & 4.067244 \\
\hline Red & 0.005458 & 2.804275 & 0.002450 & 1.221698 & 0.013313 & 6.889838 \\
\hline Skin & 0.003970 & 3.067356 & 0.000886 & 0.694646 & 0.005590 & 4.372881 \\
\hline Orange & 0.001528 & 1.193868 & 0.009350 & 7.312208 & 0.002032 & 1.548810 \\
\hline Brown & 0.007249 & 3.777906 & 0.003234 & 1.673550 & 0.004054 & 2.088440 \\
\hline Yellow & 0.003913 & 3.049058 & 0.006365 & 4.934431 & 0.005357 & 4.154822 \\
\hline Foliage & 0.007803 & 4.074478 & 0.002105 & 1.080393 & 0.002801 & 1.446664 \\
\hline Green & 0.006233 & 3.255593 & 0.003473 & 1.809795 & 0.004706 & 2.436179 \\
\hline Blue-green & 0.001735 & 1.345712 & 0.003852 & 3.017073 & 0.000729 & 0.556415 \\
\hline Blue & 0.004418 & 2.311566 & 0.006944 & 3.650813 & 0.012067 & 6.307394 \\
\hline Sky & 0.001386 & 1.074741 & 0.001252 & 0.985754 & 0.004973 & 3.870815 \\
\hline Purple & 0.004942 & 2.553928 & 0.003736 & 1.944588 & 0.002421 & 1.253062 \\
\hline Average & 0.004231 & 2.514918 & 0.003736 & 2.484796 & 0.005271 & 3.249380 \\
\hline
\end{tabular}

Table 2. The chromaticty errors and color reproduction errors of the proposed model

In subjective experiments, we use the predicted corresponding color images by the proposed model and the Breneman's corresponding color images according to the change of luminance level under the same D55 illuminants. These images are shown in Fig. 2. The predicted images by the proposed model were perceived the same as the corresponding color images of Breneman in all luminance conditions for the seven observers. 


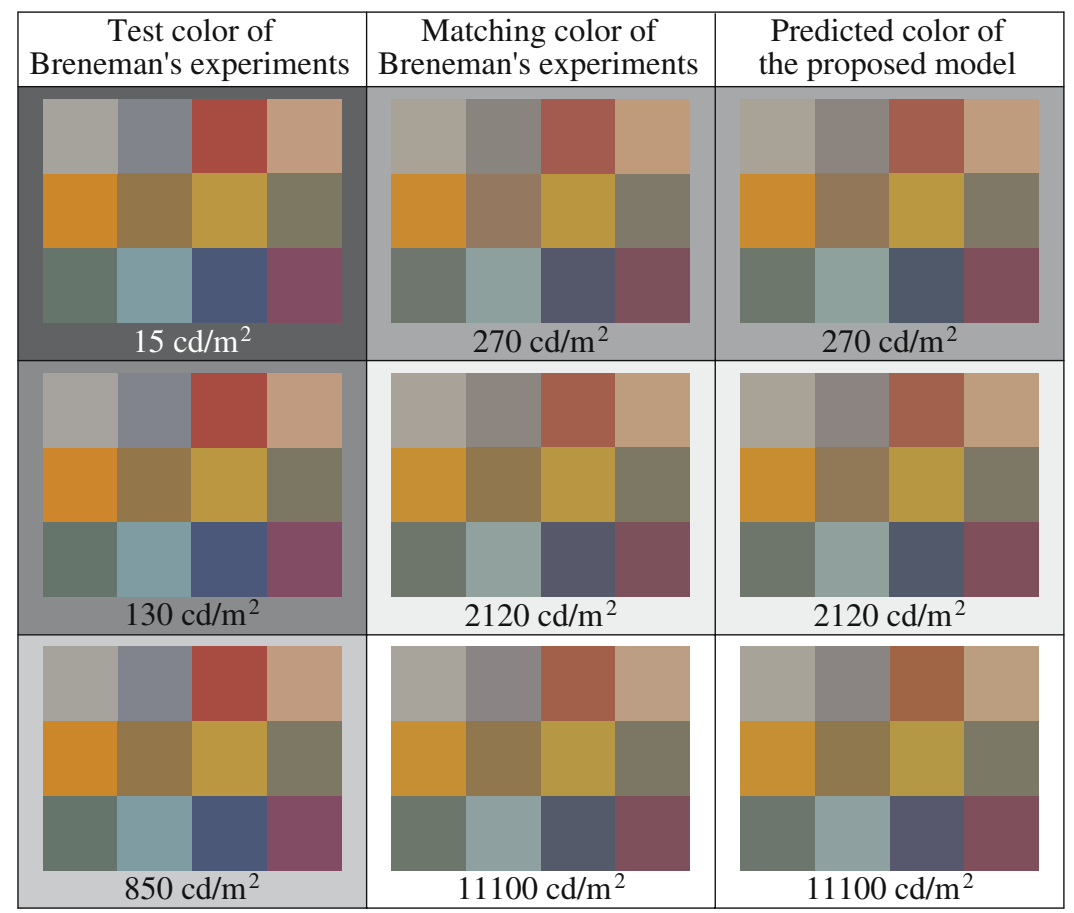

Fig. 2. Comparison between the predicted corresponding color images by the proposed model and the Breneman's corresponding color images according to the change of luminance level under the same $\mathrm{D}_{55}$ illuminants

\section{Conclusions}

In this paper, we propose the CAM for the different luminance levels under the same illuminant. The proposed model is obtained by the transform the test colors of the high luminance into the corresponding colors of the low luminance. In the proposed model, the optimal coefficients are obtained from the corresponding colors data of the Breneman's experiments. Because transfer matrix of the proposed model is the square matrix, the proposed model can be transformed easily from high luminance conditions to low luminance conditions using the inverse matrix. And once the surround viewing condition is determined, the proposed model has the coefficient of linear transformation and can be easily applied to the color display devices.

In the experimental results, we confirmed that the chromaticity errors between the predicted colors by the proposed model and the corresponding colors of the Breneman's experiments are 0.004 in $u^{\prime} v^{\prime}$ chromaticity coordinates. The prediction performance of the proposed model is excellent because these errors are the threshold value that two adjacent color patches can be distinguished. In subjective experiments, the predicted images by the proposed model were per- 
ceived the same as the corresponding color images of Breneman in all luminance conditions for the seven observers.

\section{References}

1. Hunt, R. W. G. : The Reproduction of Colour in Photography, Printing \& Television, Fountain Press, England, (1987) 177-196.

2. Fairchild, M. D. : Color Appearance Models, Addison-Wesley, New York, (1998) 173-345.

3. Wyszecki, G. and Stiles, W. S.: Color Science: Concepts and Methods, Quantitative Data and Formulae, John Wiley \& Sons, New York, (1982) 117-451.

4. MacAdam, D. L. : Color Measurement, Springer-Verlag Berlin Heidelberg, New York, (1981) 200-208.

5. Bartleson, C. J. : Comparison of chromatic-adaptation transforms, Color Res. Appl., Vol. 3, (1978) 129-136.

6. Bartleson, C. J. : Changes in color appearance with variations in chromatic adaptation, Color Res. Appl., Vol. 4, (1979) 119-138.

7. Breneman, E. J. : Corresponding chromaticities for different states of adaptation to complex visual fields, J. of Opt. Soc. Am., Vol. 4, (1987) 1115-1129.

8. Fairchild, M. D. : A model of incomplete chromatic adaptation, Proc. the 22nd Session of the CIE, Melbourne, (1991) 33-34.

9. Fairchild, M. D. : Formulation and testing of an incomplete-chromatic-adaptation model, Color Res. Appl., Vol. 16, (1991) 243-250.

10. CIE TC1-34 Final Report, The CIE 1997 Interim Colour Appearance Model (Simple Version), CIECAM97s, (1998).

11. Kim, E. S., Jang, S. W., Kwon, Y. D., Han, C. H. and Sohng, K. I.: Correspondingcolor reproduction model according to surround viewing conditions, IEICE Trans. Fund., Vol. E87-A, No. 6, (2004) 1514-1519.

12. Hunt, R. W. G. : Light and dark adaptation and the perception of color, J. of Opt. Soc. Am., Vol. 42, (1952) 190-199.

13. Hernandez-Andres, J., Romero, J. and Nieves, J. L.: Color and spectral analysis of daylight in southern Europe, J. of Opt. Soc. Am., Vol. 18, (2001) 1325-1335.

14. Bartleson, C. J. : Predicting corresponding colors with changes in adaptation, Color Res. Appl., Vol. 4, (1979) 143-155. 